\title{
ARE $Z$-BURSTS RESPONSIBLE FOR THE SUPER-GZK ULTRA HIGH ENERGY COSMIC RAYS?
}

\author{
KWANG-CHANG LAI \\ Department of Physics, National Taiwan University, Taipei, 106, Taiwan \\ kclai@phys.ntu.edu.tw \\ PISIN CHEN \\ Kavli Institute for Particle Astrophysics and Cosmology \\ Stanford Linear Accelerator Center, Stanford University, Stanford, CA 94309, USA \\ chen@slac.stanford.edu
}

Received 6 December 2005

Revised 18 January 2006

\begin{abstract}
We show that the cascade limit on ultra high energy $\operatorname{cosmic}$ neutrino (UHEC $\nu$ ) flux imposes a lower bound on the neutrino mass, provided that super-GZK events of ultra high energy cosmic rays (UHECRs) are produced from $Z$-bursts. Based on data from HiRes and AGASA, the lower bound obtained on neutrino mass violates its existing cosmological upper bound. We conclude that $Z$-bursts cannot be the dominant source for the observed super-GZK UHECR events. This is consistent with the recent ANITAlite data.
\end{abstract}

Keywords: Ultra high energy neutrinos; $Z$-burst; neutrino astronomy.

PACS No.: $98.70 . \mathrm{Sa}$

\section{Introduction}

Big bang cosmology predicts the existence of both cosmic microwave background $(\mathrm{CMB})$ and cosmic neutrino background $(\mathrm{C} \nu \mathrm{B})$. Ultra high energy cosmic protons are expected to interact with CMB photons, predominantly through the photopion production at the $\Delta$-baryon resonance, and lose their energies rapidly with an attenuation length around $50 \mathrm{Mpc}$. The ultra high energy cosmic ray (UHECR) spectrum is predicted to exhibit a cutoff — the so-called GZK cutoff ${ }^{1,2}$ — near an energy $\sim 4 \times 10^{19} \mathrm{eV}$. While observations from the HiRes experiment are consistent with the notion of a GZK cutoff, ${ }^{3}$ the AGASA data appear to suggest the opposite. ${ }^{4-7}$ This has led to speculations as to whether the GZK cutoff really exists, and, if not, what is the nature of these super-GZK events.

Existing models for super-GZK UHECRs are usually categorized into topdown and bottom-up scenarios. The top-down scenario assumes the existence of 
super-massive exotic elementary particles beyond the standard model. The major challenge of this scenario lies in the demand for a fine-tuned decay and/or annihilation rate, and, of course, the lack of any evidence for their existence. On the other hand, the bottom-up scenario, which assumes ordinary particles for the UHECRs, faces the challenge of providing an effective mechanism to accelerate particles to such ultra high energies. Even if an effective "cosmic accelerator" can be identified, the cosmic transport dictated by the GZK mechanism remains an issue, as there do not seem to exist identifiable sources within our local super-cluster $(\sim 50 \mathrm{Mpc})$ for the observed events.

To circumvent this difficulty, it was suggested that $Z$-bursts, the resonant annihilation of ultra high energy cosmic neutrino ( $\mathrm{UHEC} \nu)$ with $\mathrm{C} \nu \mathrm{B}$ into $Z$ bosons and subsequent decay into ultra high energy protons, ${ }^{8-10}$ that can occur within our local super-cluster, can account for UHECRs beyond the GZK-cutoff. ${ }^{11,12}$ With a meanfree-path comparable to the present Hubble radius, the UHEC $\nu$ serve as cosmic messengers that can avoid the GZK proton attenuation problem, without invoking physics beyond the standard model. For a $Z$-burst to take place, the UHEC $\nu$ must be at the resonant energy,

$$
E_{\mathrm{res}}=\frac{M_{Z}^{2}}{2 m_{\nu}} \cong 4 \times 10^{21}\left(\frac{1 \mathrm{eV}}{m_{\nu}}\right) \mathrm{eV},
$$

which depends on neutrino rest mass, $m_{\nu}$, and $M_{Z}$ denotes the mass of the $Z$ boson. If the $Z$-burst mechanism is indeed responsible for the observed UHECR super-GZK spectrum, then this suggests a constraint on the neutrino mass via the above relation.

Based on the $Z$-burst scenario, two groups have derived bounds on neutrino masses using AGASA data. Fodor, Katz and Ringwald ${ }^{13}$ deduce the $Z$-burst spectrum from the AGASA data by parametrizing the transition from the non-burst to the burst component near and above the "ankle" of the UHECR spectrum. Gelmini, Varieschi and Weiler ${ }^{14}$ derive their bound by requiring the $Z$-burst to produce very few events beyond the AGASA end-point energy. Our strategy, instead, is to invoke an upper limit on the UHEC $\nu$ flux so as to obtain an upper bound on the required resonant energy $E_{\text {res }}$, which can in turn be translated into a lower bound on neutrino mass.

In this paper, we derive a lower bound on neutrino mass based on the assumption that the $Z$-burst mechanism saturates the observed UHECR super-GZK spectrum. Our deduced lower bound on neutrino mass, however, turns out to be higher than the existing upper bound deduced from cosmological considerations. We thus conclude that the $Z$-burst mechanism cannot be responsible for the super-GZK UHECR spectrum. Our conclusion agrees with that from the recent ANITA-lite experiment. ${ }^{15}$

\section{UHECR Flux and $Z$-Bursts}

Assuming that all observed super-GZK UHECR proton events are induced from $Z$ bursts, the observed super-GZK proton flux must be smaller than the total $Z$-burst 
proton yield in the universe, since there must be events produced outside the local GZK-sphere $(\sim 50 \mathrm{Mpc})$ that did not reach the Earth. Furthermore, in order for $Z$-burst events to saturate the observed super-GZK spectrum, it is inevitable that there must be some $Z$-burst protons that are generated at energies below the GZKcutoff. Consequently,

$$
I_{p,>\mathrm{GZK}}^{\mathrm{obs}} \leq I_{p \mid \nu}^{Z}
$$

where $I_{p,>\text { GZK }}^{\text {obs }}$ is the total observed super-GZK proton flux with energy exceeding the GZK-cutoff and $I_{p \mid \nu}^{Z}$ is the total proton flux from $Z$-bursts, both in units of $\mathrm{cm}^{-2} \mathrm{~s}^{-1} \mathrm{sr}^{-1}$.

Although observations ${ }^{16}$ cannot completely rule out a possible contribution to the super-GZK UHECR spectrum from UHE photons, data ${ }^{16}$ suggest that protons saturate the super-GZK flux, $I_{p,>\mathrm{GZK}}^{\mathrm{obs}}=I_{>\mathrm{GZK}}^{\mathrm{obs}}$, at a confidence level of two standard deviation. Then, in terms of the total observed UHECR flux, Eq. (2) can be written as

$$
I_{>\mathrm{GZK}}^{\mathrm{obs}} \leq I_{p \mid \nu}^{Z}
$$

The AGASA experiment has accumulated 73 events above $4 \times 10^{19} \mathrm{eV}$ for a total exposure of $\sim 5.1 \times 10^{20} \mathrm{~cm}^{2} \mathrm{~s} \mathrm{sr}^{7}$ This translates into an observational super-GZK flux,

$$
I_{>\mathrm{GZK}}^{\mathrm{obs}} \simeq 1.43 \times 10^{-19} \mathrm{~cm}^{-2} \mathrm{~s}^{-1} \mathrm{sr}^{-1} .
$$

It can be shown that a power-law index of -2.78 reproduces the above flux. ${ }^{17}$

\section{Yield of $Z$-Bursts}

Now, we deduce the total $Z$-burst proton yield within a relevant cosmic volume. Solar and atmospheric data on neutrino oscillations indicate that the oscillation lengths are much shorter than the radius of the GZK-sphere. For cosmic neutrinos, the population among the three flavors should be equal. The total UHEC $\nu$ flux is therefore a factor of 3 that for a single neutrino flavor. We further assume the same flux for neutrinos and antineutrinos. By definition,

$$
I_{p \mid \nu}^{Z}=3 \xi_{p+n \mid \nu} \int_{0}^{R_{\max }} d r \int_{0}^{\infty} d E F(E, r) \sigma_{\nu \bar{\nu}}\left(E=\frac{s}{2 m_{\nu}}\right) \operatorname{Br}(Z \rightarrow \operatorname{hadrons}) n_{\nu}(r),
$$

where $F(E, r)$ is the UHEC $\nu$ flux at energy $E$ and distance $r$ from the Earth, $n_{\nu}(r)$ is the number density of the $\mathrm{C} \nu \mathrm{B}, \sigma_{\nu \bar{\nu}}(s)$ the neutrino-antineutrino cross-section at $s=2 m_{\nu} E, \operatorname{Br}(Z \rightarrow$ hadrons $)$ the branching ratio, and $\xi_{p+n \mid \nu}$ the multiplicity of nucleons per $Z$-burst.

For completeness, our integration should include all neutrinos and $Z$-burst events in the universe. Such a treatment tends to be over-conservative, as the protons deduced from $Z$-bursts outside of our local GZK-sphere will hardly survive. 
The full treatment can be found in Ref. 18. A physically reasonable, yet much simplified, calculation can be carried out by neglecting contributions from outside of our local GZK-sphere. This amounts to replacing the maximum distance $R_{\max }$ in our integration by the radius of our local GZK-sphere $\left(R_{\mathrm{GZK}} \sim 50 \mathrm{Mpc}\right)$. As the distance under consideration is much more local, all the $r$-dependence can be ignored:

$$
I_{p \mid \nu}^{Z}=3 \xi_{p+n \mid \nu} R_{\mathrm{GZK}} n_{\nu} \operatorname{Br}(Z \rightarrow \text { hadrons }) \int_{0}^{\infty} d E F(E) \sigma_{\nu \bar{\nu}}(E),
$$

where $n_{\nu}(r) \equiv n_{\nu}=112 \mathrm{~cm}^{-3}$ is the present $\mathrm{C} \nu \mathrm{B}$ neutrino-antineutrino number density per flavor.

The UHEC $\nu$ flux is commonly assumed to follow a power-law energy spectrum

$$
F(E)=F_{0} E^{-\alpha},
$$

with $F_{0}$ being a normalization factor.

Using $E=s E_{\text {res }} / M_{Z}^{2}$, we can write the integration over energy as:

$$
\int_{0}^{\infty} d E F(E) \sigma_{\nu \bar{\nu}}(s=2 m E)=E_{\mathrm{res}} \int_{0}^{\infty} \frac{d s}{M_{Z}^{2}} F\left(s E_{\mathrm{res}} / M_{Z}^{2}\right) \sigma_{\nu \bar{\nu}}(s) .
$$

As the neutrino-antineutrino annihilation cross-section is sharply peaked at the $Z$-resonance, it acts essentially like a $\delta$-function in the integration over the energy of the UHEC $\nu$ spectrum. We therefore introduce the energy-averaged cross-section ${ }^{8,12}$ :

$$
\left\langle\sigma_{\nu \bar{\nu}}\right\rangle \equiv \int \frac{d s}{M_{Z}^{2}} \sigma_{\nu \bar{\nu}}(s)=2 \pi \sqrt{2} G_{\mathrm{F}}=40.4 \mathrm{nb},
$$

which is the effective cross-section for all neutrinos within the resonance range $\left(E_{\mathrm{res}}\left(1-\Gamma_{Z} / M_{Z}\right)\right.$ to $\left.E_{\mathrm{res}}\left(1+\Gamma_{Z} / M_{Z}\right)\right)$. The integration in Eq. (8) now becomes:

$$
\begin{aligned}
E_{\mathrm{res}} \int_{0}^{\infty} \frac{d s}{M_{Z}^{2}} F\left(s E_{\mathrm{res}} / M_{Z}^{2}\right) \sigma_{\nu \bar{\nu}}(s) & \simeq F_{0} E_{\mathrm{res}}^{1-\alpha} \int_{0}^{\infty} \frac{d s}{M_{Z}^{2}} \sigma_{\nu \bar{\nu}}(s) \\
& =F_{0} E_{\mathrm{res}}^{1-\alpha}\left\langle\sigma_{\nu \bar{\nu}}\right\rangle .
\end{aligned}
$$

Putting everything together, we find

$$
I_{p \mid \nu}^{Z}=3 R_{\mathrm{GZK}} n_{\nu} \xi_{p+n \mid \nu} F_{0}\left(\frac{M_{Z}^{2}}{2 m_{\nu}}\right)^{1-\alpha}\left\langle\sigma_{\nu \bar{\nu}}\right\rangle \operatorname{Br}(Z \rightarrow \text { hadrons }) .
$$

The latest branching ratio is ${ }^{19}$ :

$$
\operatorname{Br}(Z \rightarrow \text { hadrons })=(69.91 \pm 0.07) \% .
$$

The final proton multiplicity per $Z$-burst was calculated by Fodor, Katz and Ringwald (see Ref. 20 and references therein) as a function of proton momentum, and by Gelmini, Varieschi and Weiler, ${ }^{14}$ using the event generator PYTHIA. ${ }^{21}$ They obtain $\xi_{p+n \mid \nu} \cong 2.04$ and 1.6 , respectively. We take the former value in this paper. 
Inserting numerical values of the relevant parameters into Eq. (11), the proton yield of $Z$-bursts is

$$
I_{p \mid \nu}^{Z}=2.90 \times 10^{-3} F_{0}\left(\frac{M_{Z}^{2}}{2 m_{\nu}}\right)^{1-\alpha}
$$

\section{Cascade Limit and Bound on Neutrino Mass}

We now invoke the cascade limit to constrain the UHEC $\nu$ flux. ${ }^{22,23}$ This is permissible due to the fact that neutrino productions must always be accompanied by photons and electrons. The cascades are induced as these photons or electrons interact with low energy background radiation, such as the CMB in extra galactic space and the infrared radiation within our galaxy. Any produced photons cascade down further, and eventually pile up in the energy range of $10 \mathrm{MeV}-100 \mathrm{GeV}$, with a spectrum $\propto E^{-2}$, which is consistent with the EGRET observation. ${ }^{24}$ The estimated average energy density in this range is $\omega_{\text {EGRET }} \approx 3 \times 10^{-6} \mathrm{eV} / \mathrm{cm}^{3}$. This provides an upper bound on the UHE neutrino flux:

$$
E^{2} F(E)<\frac{c}{4 \pi} \omega_{\text {cas }}<\frac{c}{4 \pi} \omega_{\text {EGRET }} .
$$

We do not assume the exact value of $\alpha=2$ for the power-law index, but instead leave $\alpha$ as a free parameter, knowing that its value should be close to 2 . Thus the parameter $F_{0}$ takes on an upper bound as follows:

$$
\begin{aligned}
& F_{0}<\frac{c}{4 \pi} \omega_{\text {EGRET }} / E_{\min }^{2-\alpha}, \quad \alpha \geq 2, \\
& F_{0}<\frac{c}{4 \pi} \omega_{\text {EGRET }} / E_{\max }^{2-\alpha}, \quad \alpha<2,
\end{aligned}
$$

where $E_{\max }$ and $E_{\min }$ are the maximum and minimum energies of the UHEC $\nu$ spectrum. Fixing the normalization factor renders an upper limit on the proton yield

$$
\begin{array}{ll}
I_{p \mid \nu}^{Z}<13.9\left(\frac{E_{\mathrm{res}}}{E_{\mathrm{min}}}\right)^{2-\alpha}\left(\frac{2 m_{\nu}}{M_{Z}^{2}}\right), \quad \alpha \geq 2, \\
I_{p \mid \nu}^{Z}<13.9\left(\frac{E_{\mathrm{res}}}{E_{\mathrm{max}}}\right)^{2-\alpha}\left(\frac{2 m_{\nu}}{M_{Z}^{2}}\right), \quad \alpha<2 .
\end{array}
$$

Implementing the cascade-limit condition, and inserting all the relevant physical quantities discussed in the previous section, Eq. (3) becomes

$$
\begin{aligned}
& m_{\nu}>28.7\left(\frac{E_{\mathrm{res}}}{E_{\min }}\right)^{\alpha-2} \mathrm{eV}, \quad \alpha \geq 2, \\
& m_{\nu}>28.7\left(\frac{E_{\max }}{E_{\mathrm{res}}}\right)^{2-\alpha} \mathrm{eV}, \quad \alpha<2 .
\end{aligned}
$$

Not all of the dependence on $m_{\nu}$ in this expression is contained in the left-hand side (LHS), as $E_{\text {res }}$ also clearly depends on $m_{\nu}$. Nevertheless, this expression has an advantage in that by definition $E_{\text {res }} / E_{\min } \geq 1$ and $E_{\max } / E_{\text {res }} \geq 1$. 
An explicit lower bound on $m_{\nu}$ can be obtained by moving all the $m_{\nu}$ dependence to the LHS. We then find:

$$
\begin{array}{ll}
m_{\nu}>\frac{1}{2}\left(\mathcal{A}^{\frac{1}{\alpha-1}} E_{\min }^{\frac{2-\alpha}{\alpha-1}} M_{Z}^{2}\right), & \alpha \geq 2, \\
m_{\nu}>\frac{1}{2}\left(\mathcal{A}^{\frac{1}{\alpha-1}} E_{\max }^{\frac{2-\alpha}{\alpha-1}} M_{Z}^{2}\right), & \alpha<2,
\end{array}
$$

where

$$
\mathcal{A}=I_{>\mathrm{GZK}}^{\mathrm{obs}}\left[\frac{c}{4 \pi} \omega_{\mathrm{EGRET}} n_{\nu} R_{\mathrm{GZK}} \xi_{p+n \mid \nu}\left\langle\sigma_{\nu \bar{\nu}}\right\rangle \operatorname{Br}(Z \rightarrow \text { hadrons })\right]^{-1} .
$$

The mass bound depends on the power-law index $\alpha$ and the values of $E_{\max }$ and $E_{\text {min }}$. Our limited knowledge on the UHEC $\nu$ renders large uncertainty in the determination of $E_{\max }$ and $E_{\min }$. One thing that is certain, however, is that the resonant energy must lie between $E_{\max }$ and $E_{\min }$ for $Z$-bursts to take place. Equation (17) indicates that the minimum value of our bound corresponds to the situation where either $E_{\max }$ or $E_{\min }$ equals $E_{\text {res }}$, or $\alpha=2$. Since we are interested in the smallest possible lower bound, we put $E_{\text {res }}=E_{\max }=E_{\min }$ in our estimate, and arrive at a lower bound for neutrino mass of:

$$
m_{\nu}>28.7_{-10.6}^{+11.8} \mathrm{eV} \quad\left(R_{\max }=R_{\mathrm{GZK}} \sim 50 \mathrm{Mpc}\right),
$$

where the error comes from fitting the AGASA data. ${ }^{17}$

Recent WMAP ${ }^{25}$ measurements of the CMB fluctuations have deduced a strong upper limit on neutrino masses, $\Sigma_{i} m_{\nu_{i}}<0.69 \mathrm{eV}$. Since any single neutrino mass of $\sim 0.04 \mathrm{eV}$ implies a near mass-degeneracy for all three active neutrinos, one concludes $m_{\nu}<0.23 \mathrm{eV}$. Two analyses ${ }^{26,27}$ that include data from WMAP, $2 \mathrm{dF}$, SDSS, and galaxy cluster surveys have arrived at a bound of $\Sigma_{i} m_{\nu_{i}} \lesssim 0.7 \mathrm{eV}$. Another analysis ${ }^{28}$ using CMB and LSS data gives $\Sigma_{i} m_{\nu_{i}} \lesssim 1 \mathrm{eV}$, but finds a stronger bound $\Sigma_{i} m_{\nu_{i}} \lesssim 0.6 \mathrm{eV}$ when priors from supernova data and Hubble Key Project are included. These newer results are close to the original WMAP bound. All these analyses converge to a cosmological upper bound of $m_{\nu} \lesssim 0.23 \mathrm{eV}$, which is 2 orders of magnitudes smaller than the lowest bound we have derived.

\section{Implications}

Our derivation is based on two assumptions: the saturation of the observed superGZK UHECR flux by the $Z$-burst mechanism and the cascade upper limit on the maximum $\mathrm{UHE} \nu$ flux.

Since the cascade limit is deduced from the cascades of photons that accompany neutrino production, it should be valid for all sources. Not only astrophysical accelerators (e.g., GRBs, AGNs, SNe, etc.) but also top-down sources, such as topological defects, superheavy $X$ particles, dark matter, etc., are all contributing to this limit, as long as photons are co-produced along with neutrinos. It is therefore generally believed ${ }^{29,30}$ that the cascade limit on $\mathrm{UHE} \nu$ flux is quite robust. 
Even under the most conservative assumption, $E_{\min }=E_{\max }=E_{\text {res }}$, i.e. that the UHEC $\nu$ spectrum is a $\delta$-function, our deduced lower bound on neutrino mass is more than 120 times larger than the existing upper bound. We therefore conclude that the $Z$-burst scenario cannot account for the observed super-GZK UHECR flux. Assuming all the parameters are fixed, our lower bound on neutrino mass can be reduced if we allow $Z$-bursts to contribute only partially to the observed UHECR flux. Based on our values, we can conclude that the $Z$-burst contribution to UHECR cannot be more than $\sim 1 \%$ within our local GZK-sphere. The recent ANITA-lite experiment ${ }^{15}$ indicates that the $Z$-burst can at best contribute $\sim 10 \%$ to the UHECR spectrum, which is consistent with our conclusion.

Analogous to the GZK process, $Z$-burst is one of the few robust cosmic interaction processes based on the standard model of particle physics. With a mean-freepath comparable to the present Hubble radius, it provides much hope to resolve the existing challenge of the bottom-up scenario. Our negative conclusion on its viability as a solution to the super-GZK puzzle seems to force us back to the original dilemma. If the excess super-GZK flux is verified, the need for a solution to puzzle will remain a challenge.

\section{Acknowledgments}

We thank P. Blasi, Je-An Gu and K. Reil for valuable discussions. K.C.L. appreciates guidance and support of W-Y. P. Hwang. This work was supported by the National Science Council (NSC 94-2112-M-002-029; NSC 93-2112-M-002-025) of Taiwan, R.O.C., and in part by US Department of Energy under Contract No. DEAC03-76SF00515.

\section{References}

1. K. Greisen, Phys. Rev. Lett. 16, 748 (1966).

2. G. T. Zatsepin and V. A. Kuzmin, Sov. Phys. JETP Lett. 4, 78 (1966).

3. R. U. Abbasi et al., Phys. Rev. Lett. 92, 151101 (2004).

4. M. Takeda et al., Phys. Rev. Lett. 81, 1163 (1998).

5. M. Takeda et al., Astropart. Phys. 19, 447 (2003).

6. N. Hayashida et al., Astron. J. 120, 2190 (2000).

7. M. Takeda et al., Astropart. Phys. 19, 447 (2003).

8. T. J. Weiler, Phys. Rev. Lett. 49, 234 (1982).

9. E. Roulet, Phys. Rev. D 47, 5247 (1993).

10. S. Yoshida, H. Dai, C. C. H. Jui and P. Sommers, Astrophys. J. 479, 547 (1997).

11. D. Fargion, B. Mele and A. Salis, Astrophys. J. 517, 725 (1999).

12. T. J. Weiler, Astropart. Phys. 11, 303 (1999).

13. Z. Fodor, S. D. Katz and A. Ringwald, Phys. Rev. Lett. 88, 171101 (2002).

14. G. Gelmini, G. Varieschi and T. J. Weiler, Phys. Rev. D 70, 113005 (2004).

15. P. Gorham, at PANIC05: Particles and Nuclei Int. Conf., Santa Fe, NM, October 24-28, 2005.

16. K. Shinozaki et al., Astrophys. J. 571, L117 (2002).

17. M. Nagano and A. A. A. Watson, Rev. Mod. Phys. 72, 689 (2000). 
18. K.-C. Lai, PhD Thesis, National Taiwan University, Janurary 2006.

19. Particle Data Group (S. Eidelman et al.), Phys. Lett. B 59, 1 (2004).

20. Z. Fodor, S. D. Katz and A. Ringwald, JHEP 06, 046 (2002).

21. T. Sjostrand, Comput. Phys. Commun. 82, 74 (1994).

22. V. S. Berezinsky, S. V. Bulanov, V. A. Dogiel, V. L. Ginzburg and V. S. Ptuskin, Astrophysics of Cosmic Rays (North-Holland, 1990).

23. V. Berezinsky and A. Smirnov, Ap. Sp. Sci. 32, 461 (1975).

24. EGRET Collab. (P. Sreekumar et al.), Astrophys. J. 494, 523 (1998).

25. WMAP Collab. (D. N. Spergel et al.), Astrophys. J. Suppl. 148, 175 (2003).

26. V. Barger, D. Marfatia and A. Tregre, Phys. Lett. B 595, 55 (2004).

27. S. W. Allen, R. W. Schmidt and S. L. Bridle, Mon. Not. Roy. Astron. Soc. 346, 593 (2003).

28. P. Crotty, J. Lesgourgues and S. Pastor, Phys. Rev. D 69, 123007 (2004).

29. Z. Fodor, S. D. Katz, A. Ringwald and H. Tu, J. Cosmol. Astropart. Phys. 0311, 015 (2003).

30. V. Berezinsky, astro-ph/0505220. 
Copyright of Modern Physics Letters $A$ is the property of World Scientific Publishing Company and its content may not be copied or emailed to multiple sites or posted to a listserv without the copyright holder's express written permission. However, users may print, download, or email articles for individual use. 